\title{
Existence of Pseudo Almost Automorphic Solutions to a Nonautonomous Heat Equation
}

\author{
Toka Diagana \\ Department of Mathematics, \\ Howard University, \\ 2441 6th Street NW, Washington, DC 20005 - USA. \\ email: tokadiag@gmail.com
}

\begin{abstract}
In this paper, upon making some suitable assumptions, we obtain the existence of pseudo-almost automorphic solutions to a nonautonomous heat equation with gradient coefficients.
\end{abstract}

\section{RESUMEN}

En este trabajo, al hacer algunos supuestos adecuados, se obtiene la existencia de pseudo soluciones automorfas a una ecuacin del calor no autnoma con coeficientes degradados.

Keywords: Pseudo almost periodicity; almost automorphic; pseudo almost automorphic; $\mathbf{S}^{p_{-}}$ pseudo almost automorphic; $\mathbf{S}^{p}$-almost automorphic; $\mathbf{S}^{p}$-pseudo almost periodic; Acquistapace and Terreni conditions; intermediate space; exponential dichotomy.

AMS Subject Classification: 43A60; 34G20.

\section{Introduction}

Fix $p>1$ and $\alpha, \beta \in \mathbf{R}$ with $0 \leq \alpha<\beta<1$. This paper is concerned with the existence of pseudoalmost automorphic solutions to the the class of abstract nonautonomous differential equations given by

$$
\frac{d}{d t}[u(t)+f(t, B u(t))]=A(t) u(t)+g(t, C u(t)), \quad t \in \mathbf{R}
$$


where $A(t)$ for $t \in \mathbf{R}$ is a family of closed linear operators on their domains $D(A(t))$ satisfying the well-known Acquistapace-Terreni conditions, $B, C$ are (possibly unbounded) linear operators, and $f: \mathbf{R} \times \mathbf{X} \mapsto \mathbf{X}_{\beta}^{t}$ and $g: \mathbf{R} \times \mathbf{X} \mapsto \mathbf{X}$ are $\mathbf{S}^{p}$-pseudo-almost automorphic functions in $t \in \mathbf{R}$ uniformly in the second variable. In view of above, there exists an evolution family $\mathcal{U}=\{U(t, s)\}_{t \geq s}$ associated with the family of operators $A(t)$. Assuming that $\mathcal{U}=\{U(t, s)\}_{t \geq s}$ is exponentially dichotomic (hyperbolic) and under some additional assumptions, it will be shown that Eq. (1.1) has a unique pseudo-almost automorphic solution. It is worth mentioning that the main result of this paper (Theorem 5.5) generalizes, to some extent, most of known results on (pseudo) almost automorphic solutions to autonomous and nonautonomous differential equations, especially those in $[9,22,48]$.

Let $\Omega \subset \mathbf{R}^{N}(N \geq 1)$ be a bounded subset with regular boundary $\Gamma=\partial \Omega$ and let $\mathbf{X}=L^{2}(\Omega)$ be the space of square integrable functions equipped with its natural topology. To illustrate our main result, we study the existence of pseudo-almost automorphic solutions to the nonautonomous heat equation with gradient coefficients given by

$$
\begin{cases}\frac{\partial}{\partial t}[\varphi+F(t, \nabla \varphi)]=a(t, x) \Delta \varphi+G(t, \nabla \varphi), & \text { in } \mathbf{R} \times \Omega \\ \varphi=0, & \text { on } \mathbf{R} \times \Gamma\end{cases}
$$

where the diffusion coefficient $a: \mathbf{R} \times \Omega \mapsto \mathbf{R}$, and $F, G: \mathbf{R} \times \mathbf{X}_{1 / 2} \mapsto L^{2}(\Omega)$ are $\mathbf{S}^{p}$-pseudo-almost automorphic functions.

The concept of pseudo-almost automorphy, which is the central tool here, was introduced in the literature a few years ago by Liang et al. [34, 47] and is a generalization of both the classical almost automorphy due to Bochner [8] and that of pseudo almost periodicity due to Zhang [18]. Such a concept has recently generated several developments and extensions. For the most recent developments, we refer the reader to [17, 25, 34, 47, 35]. More recently, in Diagana [17], the concept of $\mathbf{S}^{p}$-pseudo almost automorphy (or Stepanov-like pseudo almost automorphy) was introduced, which in fact is a natural generalization of the notion of pseudo almost automorphy.

In this paper, we make extensive use of the concept of $\mathbf{S}^{p}$-pseudo almost automorphy combined with intermediate space techniques to investigate the existence of pseudo-almost automorphic solutions Eq. (1.1) and then to the $N$-dimensional heat equation Eq. (1.2). The literature related to those intermediate spaces is very extensive, in particular, we refer the reader to the excellent book by Lunardi [33], which contains a comprehensive presentation on that topic and related issues.

Existence results related to almost periodic, asymptotically almost periodic, pseudo almost periodic and almost automorphic solutions to Eq. (1.1) in the autonomous case have recently been established in [19], [20], [22], [23], [28], [29], and [30], respectively. Though to the best of our knowledge, the existence of pseudo almost automorphic solutions to Eq. (1.1) in the case when the coefficients $F, G$ are $\mathbf{S}^{p}$-pseudo almost automorphic is an untreated original problem, which 
constitutes one of the main motivations of the present paper.

\section{Preliminaries}

Let $(\mathbf{X},\|\cdot\|),\left(\mathbf{Y},\|\cdot\|_{\mathbf{Y}}\right)$ be two Banach spaces. Let $B C(\mathbf{R}, \mathbf{X})$ (respectively, $\left.B C(\mathbf{R} \times \mathbf{Y}, \mathbf{X})\right)$ denote the collection of all $\mathbf{X}$-valued bounded continuous functions (respectively, the class of jointly bounded continuous functions $F: \mathbf{R} \times \mathbf{Y} \mapsto \mathbf{X})$. The space $B C(\mathbf{R}, \mathbf{X})$ equipped with the sup norm $\|\cdot\|_{\infty}$ is a Banach space.

Furthermore, $C(\mathbf{R}, \mathbf{Y})$ (respectively, $C(\mathbf{R} \times \mathbf{Y}, \mathbf{X})$ ) denotes the class of continuous functions from $\mathbf{R}$ into $\mathbf{Y}$ (respectively, the class of jointly continuous functions $F: \mathbf{R} \times \mathbf{Y} \mapsto \mathbf{X}$ ).

The notation $B(\mathbf{X}, \mathbf{Y})$ stands for the Banach space of bounded linear operators from $\mathbf{X}$ into $\mathbf{Y}$ equipped with its natural topology; in particular, this is simply denoted $B(\mathbf{X})$ whenever $\mathbf{X}=\mathbf{Y}$.

Definition 2.1. [41] The Bochner transform $f^{b}(t, s), t \in \mathbf{R}, s \in[0,1]$ of a function $f: \mathbf{R} \mapsto \mathbf{X}$ is defined by $f^{b}(t, s):=f(t+s)$.

Remark 2.2. (i) A function $\varphi(t, s), t \in \mathbf{R}, s \in[0,1]$, is the Bochner transform of a certain function $f, \varphi(t, s)=f^{b}(t, s)$, if and only if $\varphi(t+\tau, s-\tau)=\varphi(s, t)$ for all $t \in \mathbf{R}, s \in[0,1]$ and $\tau \in[s-1, s]$.

(ii) Note that if $f=h+\varphi$, then $f^{b}=h^{b}+\varphi^{b}$. Moreover, $(\lambda f)^{b}=\lambda f^{b}$ for each scalar $\lambda$.

Definition 2.3. The Bochner transform $F^{b}(t, s, u), t \in \mathbf{R}, s \in[0,1], u \in \mathbf{X}$ of a function $F(t, u)$ on $\mathbf{R} \times \mathbf{X}$, with values in $\mathbf{X}$, is defined by

$$
F^{b}(t, s, u):=F(t+s, u)
$$

for each $u \in \mathbf{X}$.

Definition 2.4. Let $p \in[1, \infty)$. The space $B S^{p}(\mathbf{X})$ of all Stepanov bounded functions, with the exponent $p$, consists of all measurable functions $f: \mathbf{R} \mapsto \mathbf{X}$ such that $f^{b}$ belongs to $L^{\infty}\left(\mathbf{R} ; L^{p}((0,1), \mathbf{X})\right)$. This is a Banach space with the norm

$$
\|f\|_{S^{p}}:=\left\|f^{b}\right\|_{L^{\infty}\left(\mathbf{R}, L^{p}\right)}=\sup _{t \in \mathbf{R}}\left(\int_{t}^{t+1}\|f(\tau)\|^{p} d \tau\right)^{1 / p} .
$$

\section{1 $\quad \mathrm{S}^{p}$-Pseudo Almost Periodicity}

Definition 2.5. A function $f \in C(\mathbf{R}, \mathbf{X})$ is called (Bohr) almost periodic if for each $\varepsilon>0$ there exists $l(\varepsilon)>0$ such that every interval of length $l(\varepsilon)$ contains a number $\tau$ with the property that

$$
\|f(t+\tau)-f(t)\|<\varepsilon \text { for each } t \in \mathbf{R} .
$$

The number $\tau$ above is called an $\varepsilon$-translation number of $f$, and the collection of all such functions will be denoted $A P(\mathbf{X})$. 
Definition 2.6. A function $F \in C(\mathbf{R} \times \mathbf{Y}, \mathbf{X})$ is called (Bohr) almost periodic in $t \in \mathbf{R}$ uniformly in $y \in K$ where $K \subset \mathbf{Y}$ is any compact subset $K \subset \mathbf{Y}$ if for each $\varepsilon>0$ there exists $l(\varepsilon)$ such that every interval of length $l(\varepsilon)$ contains a number $\tau$ with the property that

$$
\|F(t+\tau, y)-F(t, y)\|<\varepsilon \text { for each } t \in \mathbf{R}, \quad y \in K
$$

The collection of those functions is denoted by $A P(\mathbf{R} \times \mathbf{Y})$.

Define the classes of functions $P A P_{0}(\mathbf{X})$ and $P A P_{0}(\mathbf{R} \times \mathbf{X})$ respectively as follows:

$$
P A P_{0}(\mathbf{X}):=\left\{u \in B C(\mathbf{R}, \mathbf{X}): \lim _{T \rightarrow \infty} \frac{1}{2 T} \int_{-T}^{T}\|u(s)\| d s=0\right\},
$$

and $P A P_{0}(\mathbf{R} \times \mathbf{Y})$ is the collection of all functions $F \in B C(\mathbf{R} \times \mathbf{Y}, \mathbf{X})$ such that

$$
\lim _{T \rightarrow \infty} \frac{1}{2 T} \int_{-T}^{T}\|F(t, u)\| d t=0
$$

uniformly in $u \in \mathbf{Y}$.

Definition 2.7. [18] A function $f \in B C(\mathbf{R}, \mathbf{X})$ is called pseudo almost periodic if it can be expressed as $f=h+\varphi$, where $h \in A P(\mathbf{X})$ and $\varphi \in P A P_{0}(\mathbf{X})$. The collection of such functions will be denoted by $P A P(\mathbf{X})$.

Definition 2.8. [18] A function $F \in C(\mathbf{R} \times \mathbf{Y}, \mathbf{X})$ is said to be pseudo almost periodic if it can be expressed as $F=G+\Phi$, where $G \in A P(\mathbf{R} \times \mathbf{Y})$ and $\phi \in P A P_{0}(\mathbf{R} \times \mathbf{Y})$. The collection of such functions will be denoted by $P A P(\mathbf{R} \times \mathbf{Y})$.

Define $A A_{0}(\mathbf{R} \times \mathbf{Y})$ as the collection of all functions $F \in B C(\mathbf{R} \times \mathbf{Y}, \mathbf{X})$ such that

$$
\lim _{T \rightarrow \infty} \frac{1}{2 T} \int_{-T}^{T}\|F(t, u)\| d t=0
$$

uniformly in $u \in K$, where $K \subset \mathbf{Y}$ is any bounded subset.

Obviously,

$$
P A P_{0}(\mathbf{R} \times \mathbf{Y}) \subset A A_{0}(\mathbf{R} \times \mathbf{Y})
$$

A weaker version of Definition 2.8 is the following:

Definition 2.9. A function $F \in C(\mathbf{R} \times \mathbf{Y}, \mathbf{X})$ is said to be B-pseudo almost periodic if it can be expressed as $F=G+\Phi$, where $G \in A P(\mathbf{R} \times \mathbf{Y})$ and $\phi \in A A_{0}(\mathbf{R} \times \mathbf{Y})$. The collection of such functions will be denoted by $B P A P(\mathbf{R} \times \mathbf{Y})$. 
Definition 2.10. $[15,16]$ A function $f \in B S^{p}(\mathbf{X})$ is called $\mathbf{S}^{p}$-pseudo almost periodic (or Stepanovlike pseudo almost periodic) if it can be expressed as $f=h+\varphi$, where $h^{b} \in A P\left(L^{p}((0,1), \mathbf{X})\right)$ and $\varphi^{b} \in P A P_{0}\left(L^{p}((0,1), \mathbf{X})\right)$. The collection of such functions will be denoted by $P A P^{p}(\mathbf{X})$.

In other words, a function $f \in L_{l o c}^{p}(\mathbf{R}, \mathbf{X})$ is said to be $\mathbf{S}^{p}$-pseudo almost periodic if its Bochner transform $f^{b}: \mathbf{R} \rightarrow L^{p}((0,1), \mathbf{X})$ is pseudo almost periodic in the sense that there exist two functions $h, \varphi: \mathbf{R} \mapsto \mathbf{X}$ such that $f=h+\varphi$, where $h^{b} \in A P\left(L^{p}((0,1), \mathbf{X})\right)$ and $\varphi^{b} \in P A P_{0}\left(L^{p}((0,1), \mathbf{X})\right)$.

To define the notion of $S^{p}$-pseudo almost automorphy for functions of the form $F: \mathbf{R} \times \mathbf{Y} \mapsto \mathbf{Y}$, we need to define the $\mathbf{S}^{p}$-pseudo almost periodicity for these functions as follows:

Definition 2.11. A function $F: \mathbf{R} \times \mathbf{Y} \mapsto \mathbf{X},(t, u) \mapsto F(t, u)$ with $F(\cdot, u) \in L_{l o c}^{p}(\mathbf{R}, \mathbf{X})$ for each $u \in \mathbf{X}$, is said to be $\mathbf{S}^{p}$-pseudo almost periodic if there exist two functions $H, \Phi: \mathbf{R} \times \mathbf{Y} \mapsto \mathbf{X}$ such that $F=H+\Phi$, where $H^{b} \in A P\left(\mathbf{R} \times L^{p}((0,1), \mathbf{X})\right)$ and $\Phi^{b} \in A A_{0}\left(\mathbf{R} \times L^{p}((0,1), \mathbf{X})\right)$.

The collection of those $\mathbf{S}^{p}$-pseudo almost periodic functions $F: \mathbf{R} \times \mathbf{Y} \mapsto \mathbf{X}$ will be denoted $P A P^{p}(\mathbf{R} \times \mathbf{Y})$.

\section{$2.2 \quad \mathrm{~S}^{p}$-Almost Automorphy}

The notion of $\mathbf{S}^{p}$-almost automorphy is a new notion due to N'Guérékata and Pankov [40].

Definition 2.12. (Bochner) A function $f \in C(\mathbf{R}, \mathbf{X})$ is said to be almost automorphic if for every sequence of real numbers $\left(s_{n}^{\prime}\right)_{n \in \mathbb{N}}$, there exists a subsequence $\left(s_{n}\right)_{n \in \mathbb{N}}$ such that

$$
g(t):=\lim _{n \rightarrow \infty} f\left(t+s_{n}\right)
$$

is well defined for each $t \in \mathbf{R}$, and

$$
\lim _{n \rightarrow \infty} g\left(t-s_{n}\right)=f(t)
$$

for each $t \in \mathbf{R}$.

Remark 2.13. The function $g$ in Definition 2.12 is measurable, but not necessarily continuous. Moreover, if $g$ is continuous, then $f$ is uniformly continuous. If the convergence above is uniform in $t \in \mathbf{R}$, then $f$ is almost periodic. Denote by $A A(\mathbf{X})$ the collection of all almost automorphic functions $\mathbf{R} \rightarrow \mathbf{X}$. Note that $A A(\mathbf{X})$ equipped with the sup norm, $\|\cdot\|_{\infty}$, turns out to be a Banach space.

We will denote by $A A_{u}(\mathbf{X})$ the closed subspace of all functions $f \in A A(\mathbf{X})$ with $g \in C(\mathbf{R}, \mathbf{X})$. Equivalently, $f \in A A_{u}(\mathbf{X})$ if and only if $f$ is almost automorphic and the convergence in Definition 2.12 are uniform on compact intervals, i.e. in the Fréchet space $C(\mathbf{R}, \mathbf{X})$. Indeed, if $f$ is almost automorphic, then, its range is relatively compact. Obviously, the following inclusions hold:

$$
A P(\mathbf{X}) \subset A A_{u}(\mathbf{X}) \subset A A(\mathbf{X}) \subset B C(\mathbf{X}) .
$$


Definition 2.14. [40] The space $A S^{p}(\mathbf{X})$ of Stepanov-like almost automorphic functions (or $\mathbf{S}^{p}$ almost automorphic) consists of all $f \in B S^{p}(\mathbf{X})$ such that $f^{b} \in A A\left(L^{p}(0,1 ; \mathbf{X})\right)$. That is, a function $f \in L_{l o c}^{p}(\mathbf{R} ; \mathbf{X})$ is said to be $\mathbf{S}^{p}$-almost automorphic if its Bochner transform $f^{b}: \mathbf{R} \rightarrow$ $L^{p}(0,1 ; \mathbf{X})$ is almost automorphic in the sense that for every sequence of real numbers $\left(s_{n}^{\prime}\right)_{n \in \mathbb{N}}$, there exists a subsequence $\left(s_{n}\right)_{n \in \mathbb{N}}$ and a function $g \in L_{l o c}^{p}(\mathbf{R} ; \mathbf{X})$ such that

$$
\begin{gathered}
{\left[\int_{t}^{t+1}\left\|f\left(s_{n}+s\right)-g(s)\right\|^{p} d s\right]^{1 / p} \rightarrow 0, \text { and }} \\
{\left[\int_{t}^{t+1}\left\|g\left(s-s_{n}\right)-f(s)\right\|^{p} d s\right]^{1 / p} \rightarrow 0}
\end{gathered}
$$

as $n \rightarrow \infty$ pointwise on $\mathbf{R}$.

Remark 2.15. It is clear that if $1 \leq p<q<\infty$ and $f \in L_{l o c}^{q}(\mathbf{R} ; \mathbf{X})$ is $\mathbf{S}^{q}$-almost automorphic, then $f$ is $\mathbf{S}^{p}$-almost automorphic. Also if $f \in A A(\mathbf{X})$, then $f$ is $\mathbf{S}^{p}$-almost automorphic for any $1 \leq p<\infty$. Moreover, it is clear that $f \in A A_{u}(\mathbf{X})$ if and only if $f^{b} \in A A\left(L^{\infty}(0,1 ; \mathbf{X})\right)$. Thus, $A A_{u}(\mathbf{X})$ can be considered as $A S^{\infty}(\mathbf{X})$.

Definition 2.16. A function $F: \mathbf{R} \times \mathbf{Y} \mapsto \mathbf{X},(t, u) \mapsto F(t, u)$ with $F(\cdot, u) \in L_{l o c}^{p}(\mathbf{R} ; \mathbf{X})$ for each $u \in \mathbf{Y}$, is said to be $\mathbf{S}^{p}$-almost automorphic in $t \in \mathbf{R}$ uniformly in $u \in \mathbf{Y}$ if $t \mapsto F(t, u)$ is $\mathbf{S}^{p}$-almost automorphic for each $u \in \mathbf{Y}$, that is, for every sequence of real numbers $\left(s_{n}^{\prime}\right)_{n \in \mathbb{N}}$, there exists a subsequence $\left(s_{n}\right)_{n \in \mathbb{N}}$ and a function $G(\cdot, u) \in L_{l o c}^{p}(\mathbf{R} ; \mathbf{X})$ such that

$$
\begin{gathered}
{\left[\int_{t}^{t+1}\left\|F\left(s_{n}+s, u\right)-G(s, u)\right\|^{p} d s\right]^{1 / p} \rightarrow 0, \text { and }} \\
{\left[\int_{t}^{t+1}\left\|G\left(s-s_{n}, u\right)-F(s, u)\right\|^{p} d s\right]^{1 / p} \rightarrow 0}
\end{gathered}
$$

as $n \rightarrow \infty$ pointwise on $\mathbf{R}$ for each $u \in \mathbf{Y}$.

The collection of those $\mathbf{S}^{p}$-almost automorphic functions $F: \mathbf{R} \times \mathbf{Y} \mapsto \mathbf{X}$ will be denoted by $A S^{p}(\mathbf{R} \times \mathbf{Y})$.

\subsection{Pseudo Almost Automorphy}

The notion of pseudo almost automorphy is a new notion due to Liang, Xiao and Zhang [47, 35].

Definition 2.17. A function $f \in C(\mathbf{R}, \mathbf{X})$ is called pseudo almost automorphic if it can be expressed as $f=h+\varphi$, where $h \in A A(\mathbf{X})$ and $\varphi \in P A P_{0}(\mathbf{X})$. The collection of such functions will be denoted by $P A A(\mathbf{X})$.

Obviously, the following inclusions hold:

$$
A P(\mathbf{X}) \subset P A P(\mathbf{X}) \subset P A A(\mathbf{X}) \text { and } A P(\mathbf{X}) \subset A A(\mathbf{X}) \subset P A A(\mathbf{X}) .
$$


Definition 2.18. A function $F \in C(\mathbf{R} \times \mathbf{Y}, \mathbf{X})$ is said to be pseudo almost automorphic if it can be expressed as $F=G+\Phi$, where $G \in A A(\mathbf{R} \times \mathbf{Y})$ and $\varphi \in A A_{0}(\mathbf{R} \times \mathbf{Y})$. The collection of such functions will be denoted by $P A A(\mathbf{R} \times \mathbf{Y})$.

A substantial result is the next theorem, which is due to Liang et al. [47].

Theorem 2.19. [47] The space PAA(X) equipped with the sup norm $\|\cdot\|_{\infty}$ is a Banach space.

We also have the following composition result.

Theorem 2.20. [47]] If $f: \mathbf{R} \times \mathbf{Y} \mapsto \mathbf{X}$ belongs to $P A A(\mathbf{R} \times \mathbf{Y})$ and if $x \mapsto f(t, x)$ is uniformly continuous on any bounded subset $K$ of $\mathbf{Y}$ for each $t \in \mathbf{R}$, then the function defined by $h(t)=$ $f(t, \varphi(t))$ belongs to $P A A(\mathbf{X})$ provided $\varphi \in P A A(\mathbf{Y})$.

\section{$3 \quad \mathbf{S}^{p}$-Pseudo Almost Automorphy}

This section is devoted to the notion of $\mathbf{S}^{p}$-pseudo almost automorphy. Such a concept is completely new and is due to Diagana [17].

Definition 3.1. [17] A function $f \in B S^{p}(\mathbf{X})$ is called $\mathbf{S}^{p}$-pseudo almost automorphic (or Stepanovlike pseudo almost automorphic) if it can be expressed as

$$
f=h+\varphi,
$$

where $h^{b} \in A A\left(L^{p}((0,1), \mathbf{X})\right)$ and $\varphi^{b} \in P A P_{0}\left(L^{p}((0,1), \mathbf{X})\right)$. The collection of such functions will be denoted by $P A A^{p}(\mathbf{X})$.

Clearly, a function $f \in L_{l o c}^{p}(\mathbf{R}, \mathbf{X})$ is said to be $\mathbf{S}^{p}$-pseudo almost automorphic if its Bochner transform $f^{b}: \mathbf{R} \rightarrow L^{p}((0,1), \mathbf{X})$ is pseudo almost automorphic in the sense that there exist two functions $h, \varphi: \mathbf{R} \mapsto \mathbf{X}$ such that $f=h+\varphi$, where $h^{b} \in A A\left(L^{p}((0,1), \mathbf{X})\right)$ and $\varphi^{b} \in$ $P A P_{0}\left(L^{p}((0,1), \mathbf{X})\right)$.

Theorem 3.2. [17] If $f \in P A A(\mathbf{X})$, then $f \in P A A^{p}(\mathbf{X})$ for each $1 \leq p<\infty$. In other words, $P A A(\mathbf{X}) \subset P A A^{p}(\mathbf{X})$.

Obviously, the following inclusions hold:

$$
\begin{gathered}
A P(\mathbf{X}) \subset P A P(\mathbf{X}) \subset P A A(\mathbf{X}) \subset P A A^{p}(\mathbf{X}), \\
A P(\mathbf{X}) \subset A A(\mathbf{X}) \subset P A A(\mathbf{X}) \subset P A A^{p}(\mathbf{X}) .
\end{gathered}
$$

Theorem 3.3. [17] The space $P A A^{p}(\mathbf{X})$ equipped with the norm $\|\cdot\|_{\mathbf{S}^{p}}$ is a Banach space. 
Definition 3.4. A function $F: \mathbf{R} \times \mathbf{Y} \mapsto \mathbf{X},(t, u) \mapsto F(t, u)$ with $F(\cdot, u) \in L^{p}(\mathbf{R}, \mathbf{X})$ for each $u \in \mathbf{Y}$, is said to be $\mathbf{S}^{p}$-pseudo almost automorphic if there exists two functions $H, \Phi: \mathbf{R} \times \mathbf{Y} \mapsto \mathbf{X}$ such that

$$
F=H+\Phi,
$$

where $H^{b} \in A A\left(\mathbf{R} \times L^{p}((0,1), \mathbf{X})\right)$ and $\Phi^{b} \in A A_{0}\left(\mathbf{R} \times L^{p}((0,1), \mathbf{X})\right)$. The collection of those $\mathbf{S}^{p}$-pseudo almost automorphic functions will be denoted by $P A A^{p}(\mathbf{R} \times \mathbf{Y})$.

We have the following composition theorems.

Theorem 3.5. Let $F: \mathbf{R} \times \mathbf{X} \mapsto \mathbf{X}$ be a $S^{p}$-pseudo almost automorphic function. Suppose that $F(t, u)$ is Lipschitzian in $u \in \mathbf{X}$ uniformly in $t \in \mathbf{R}$, that is there exists $L>0$ such

$$
\|F(t, u)-F(t, v)\| \leq L .\|u-v\|
$$

for all $t \in \mathbf{R},(u, v) \in \mathbf{X} \times \mathbf{X}$.

If $\phi \in P A A^{p}(\mathbf{X})$, then $\Gamma: \mathbf{R} \rightarrow \mathbf{X}$ defined by $\Gamma(\cdot):=F(\cdot, \phi(\cdot))$ belongs to $P A A^{p}(\mathbf{X})$.

Proof. Let $F=H+\Phi$, where $H^{b} \in A A\left(\mathbf{R} \times L^{p}((0,1), \mathbf{X})\right)$ and $\Phi^{b} \in A A_{0}\left(\mathbf{R} \times L^{p}((0,1), \mathbf{X})\right)$. Similarly, let $\phi=\phi_{1}+\phi_{2}$, where $\phi_{1}^{b} \in A A\left(L^{p}((0,1), \mathbf{X})\right)$ and $\phi_{2}^{b} \in P A P_{0}\left(L^{p}((0,1), \mathbf{X})\right)$, that is,

$$
\lim _{T \rightarrow \infty} \frac{1}{2 T} \int_{-T}^{T}\left(\int_{t}^{t+1}\left\|\varphi_{2}(\sigma)\right\|^{p} d \sigma\right)^{1 / p} d t=0
$$

for all $t \in \mathbf{R}$.

It is obvious to see that $F^{b}(\cdot, \phi(\cdot)): \mathbf{R} \mapsto L^{p}((0,1), \mathbf{X})$. Now decompose $F^{b}$ as follows

$$
\begin{aligned}
F^{b}(\cdot, \phi(\cdot)) & =H^{b}\left(\cdot, \phi_{1}(\cdot)\right)+F^{b}(\cdot, \phi(\cdot))-H^{b}\left(\cdot, \phi_{1}(\cdot)\right) \\
& =H^{b}\left(\cdot, \phi_{1}(\cdot)\right)+F^{b}(\cdot, \phi(\cdot))-F^{b}\left(\cdot, \phi_{1}(\cdot)\right)+\Phi^{b}\left(\cdot, \phi_{1}(\cdot)\right)
\end{aligned}
$$

Using the theorem of composition of almost automorphic functions, it is easy to see that $H^{b}\left(\cdot, \phi_{1}(\cdot)\right) \in A A\left(L^{p}((0,1), \mathbf{X})\right)$. Now, set

$$
G^{b}(\cdot):=F^{b}(\cdot, \phi(\cdot))-F^{b}\left(\cdot, \phi_{1}(\cdot)\right) .
$$

Clearly, $G^{b}(\cdot) \in P A P_{0}\left(L^{p}((0,1), \mathbf{X})\right)$. Indeed, we have

$$
\begin{aligned}
\int_{t}^{t+1}\|G(\sigma)\|^{p} d \sigma & =\int_{t}^{t+1}\left\|F(\sigma, \phi(\sigma))-F\left(\sigma, \phi_{1}(\sigma)\right)\right\|^{p} d \sigma \\
& \leq L^{p} \int_{t}^{t+1}\left\|\phi(\sigma)-\phi_{1}(\sigma)\right\|^{p} d \sigma \\
& =L^{p} \int_{t}^{t+1}\left\|\phi_{2}(\sigma)\right\|^{p} d \sigma,
\end{aligned}
$$


and hence for $T>0$,

$$
\frac{1}{2 T} \int_{-T}^{T}\left(\int_{t}^{t+1}\|G(\sigma)\|^{p} d \sigma\right)^{1 / p} d t \leq \frac{L}{2 T} \int_{-T}^{T}\left(\int_{t}^{t+1}\left\|\phi_{2}(\sigma)\right\|^{p} d \sigma\right)^{1 / p} d t .
$$

Now using Eq. (3.2), it follows that

$$
\lim _{T \rightarrow \infty} \frac{1}{2 T} \int_{-T}^{T}\left(\int_{t}^{t+1}\|G(\sigma)\|^{p} d \sigma\right)^{1 / p} d t=0 .
$$

Using the theorem of composition of functions of $P A P\left(L^{p}((0,1), \mathbf{X})\right)$ (see [18]) it is easy to see that $\Phi^{b}\left(\cdot, \phi_{1}(\cdot)\right) \in P A P_{0}\left(L^{p}((0,1), \mathbf{X})\right)$.

Theorem 3.6. Let $F=H+\Phi: \mathbf{R} \times \mathbf{X} \mapsto \mathbf{X}$ be a $S^{p}$-pseudo almost automorphic function, where $H^{b} \in A A\left(\mathbf{R} \times L^{p}((0,1), \mathbf{X})\right)$ and $\Phi^{b} \in A A_{0}\left(\mathbf{R} \times L^{p}((0,1), \mathbf{X})\right)$. Suppose that $F(t, u)$ and $\Phi(t, x)$ are uniformly continuous in every bounded subset $K \subset \mathbf{X}$ uniformly for $t \in \mathbf{R}$. If $g \in P A A^{p}(\mathbf{X})$, then $\Gamma: \mathbf{R} \rightarrow \mathbf{X}$ defined by $\Gamma(\cdot):=F(\cdot, g(\cdot))$ belongs to $P A A^{p}(\mathbf{X})$.

Proof. Let $F=H+\Phi$, where $H^{b} \in A A\left(\mathbf{R} \times L^{p}((0,1), \mathbf{X})\right)$ and $\Phi^{b} \in A A_{0}\left(\mathbf{R} \times L^{p}((0,1), \mathbf{X})\right)$. Similarly, let $g=\phi_{1}+\phi_{2}$, where $\phi_{1}^{b} \in A A\left(L^{p}((0,1), \mathbf{X})\right)$ and $\phi_{2}^{b} \in P A P_{0}\left(L^{p}((0,1), \mathbf{X})\right)$.

It is obvious to see that $F^{b}(\cdot, g(\cdot)): \mathbf{R} \mapsto L^{p}((0,1), \mathbf{X})$. Now decompose $F^{b}$ as follows

$$
\begin{aligned}
F^{b}(\cdot, g(\cdot)) & =H^{b}\left(\cdot, \phi_{1}(\cdot)\right)+F^{b}(\cdot, g(\cdot))-H^{b}\left(\cdot, \phi_{1}(\cdot)\right) \\
& =H^{b}\left(\cdot, \phi_{1}(\cdot)\right)+F^{b}(\cdot, g(\cdot))-F^{b}\left(\cdot, \phi_{1}(\cdot)\right)+\Phi^{b}\left(\cdot, \phi_{1}(\cdot)\right) .
\end{aligned}
$$

Using the theorem of composition of almost automorphic functions, it is easy to see that $H^{b}\left(\cdot, \phi_{1}(\cdot)\right) \in A A\left(L^{p}((0,1), \mathbf{X})\right)$. Now, set

$$
G^{b}(\cdot):=F^{b}(\cdot, g(\cdot))-F^{b}\left(\cdot, \phi_{1}(\cdot)\right) .
$$

We claim that $G^{b}(\cdot) \in P A P_{0}\left(L^{p}((0,1), \mathbf{X})\right)$. First of all, note that the uniformly continuity of $F$ on bounded subsets $K \subset \mathbf{X}$ yields the uniform continuity of its Bohr transform $F^{b}$ on bounded subsets of $\mathbf{X}$. Since both $g, \phi_{1}$ are bounded functions it follows that there exists $K \subset \mathbf{X}$ a bounded subset such that $g(\sigma), \phi_{1}(\sigma) \in K$ for each $\sigma \in \mathbf{R}$. Now from the uniform continuity of $F^{b}$ on bounded subsets of $\mathbf{X}$, it obviously follows that $F^{b}$ is uniformly continuous on $K$ uniformly for each $t \in \mathbf{R}$. Therefore for every $\varepsilon>0$ there exists $\delta>0$ such that for all $X, Y \in K$ with $\|X-Y\|<\delta$ yield

$$
\left\|F^{b}(\sigma, X)-F^{b}(\sigma, Y)\right\|<\varepsilon \text { for all } \sigma \in \mathbf{R} .
$$

Using the proof of the composition theorem [47, Theorem 2.4] (applied to $F^{b}$ ) it follows

$$
\lim _{T \rightarrow \infty} \frac{1}{2 T} \int_{-T}^{T}\left(\int_{t}^{t+1}\|G(\sigma)\|^{p} d \sigma\right)^{1 / p} d t=0
$$

Using the theorem of composition [47, Theorem 2.4] for functions of $P A P_{0}\left(L^{p}((0,1), \mathbf{X})\right)$ it is easy to see that $\Phi^{b}\left(\cdot, \phi_{1}(\cdot)\right) \in P A P_{0}\left(L^{p}((0,1), \mathbf{X})\right)$. 


\section{Evolution Families}

This section is devoted to some preliminary results needed in the sequel. We basically use the same setting as in [7] with slight adjustments. Throughout the rest of this paper, $(\mathbf{X},\|\cdot\|)$ stands for a Banach space, $A(t)$ for $t \in \mathbf{R}$ is a family of closed linear operators on $\mathbf{D}=D(A(t))$, which is independent of $t$, satisfying the so-called Acquistapace and Terreni conditions (Hypothesis (H.1)). Moreover, the operators $A(t)$ are not necessarily densely defined. The linear operators $B, C$ are (possibly unbounded) defined on $\mathbf{X}$ such that $A(t)+B+C$ is not trivial for each $t \in \mathbf{R}$. The functions, $f: \mathbf{R} \times \mathbf{X} \mapsto \mathbf{X}_{\beta}^{t}(0<\alpha<\beta<1), g: \mathbf{R} \times \mathbf{X} \mapsto \mathbf{X}$ are respectively jointly continuous satisfying some additional assumptions.

If $L$ is a linear operator on the Banach space $\mathbf{X}$, then:

- $D(L)$ stands for its domain;

- $\rho(L)$ stands for its resolvent;

- $\sigma(L)$ stands for its spectrum;

- $N(L)$ stands for its null-space or kernel; and

- $R(L)$ stands for its range.

Moreover, one sets $R(\lambda, L):=(\lambda I-L)^{-1}$ for all $\lambda \in \rho(A)$. Furthermore, we set $Q=I-P$ for a projection $P$.

Hypothesis (H.1). The family of closed linear operators $A(t)$ for $t \in \mathbf{R}$ on $\mathbf{X}$ with domain $D(A(t))$ (possibly not densely defined) satisfy the so-called Acquistapace-Terreni conditions, that is, there exist constants $\omega \in \mathbf{R}, \theta \in\left(\frac{\pi}{2}, \pi\right), L>0$ and $\mu, \nu \in(0,1]$ with $\mu+\nu>1$ such that

$$
\Sigma_{\theta} \cup\{0\} \subset \rho(A(t)-\omega) \ni \lambda, \quad\|R(\lambda, A(t)-\omega)\| \leq \frac{K}{1+|\lambda|} \quad \text { for all } t \in \mathbf{R},
$$

and

$$
\|(A(t)-\omega) R(\lambda, A(t)-\omega)[R(\omega, A(t))-R(\omega, A(s))]\| \leq L|t-s|^{\mu}|\lambda|^{-\nu}
$$

for $t, s \in \mathbf{R}, \lambda \in \Sigma_{\theta}:=\{\lambda \in \mathbb{C} \backslash\{0\}:|\arg \lambda| \leq \theta\}$.

Note that in the particular case when $A(t)$ has a constant domain $\mathbf{D}=D(A(t))$, it is wellknown $[4,42]$ that Eq. (4.2) can be replaced with the following: There exist constants $L$ and $0<\mu \leq 1$ such that

$$
\|(A(t)-A(s)) R(\omega, A(r))\| \leq L|t-s|^{\mu}, s, t, r \in \mathbf{R} .
$$


It should mentioned that (H.1) was introduced in the literature by Acquistapace and Terreni in $[2,3]$ for $\omega=0$. Among other things, the Acquistapace-Terreni Conditions ensure that there exists a unique evolution family

$$
\mathcal{U}=\{U(t, s): t, s \in \mathbf{R} \text { such that } t \geq s\}
$$

on $\mathbf{X}$ associated with $A(t)$ such that $U(t, s) \mathbf{X} \subseteq D(A(t))$ for all $t, s \in \mathbf{R}$ with $t \geq s$, and

(a) $U(t, s) U(s, r)=U(t, r)$ for $t, s \in \mathbf{R}$ such that $t \geq s \geq r$;

(b) $U(t, t)=I$ for $t \in \mathbf{R}$ where $I$ is the identity operator of $\mathbf{X}$;

(c) $(t, s) \mapsto U(t, s) \in B(\mathbf{X})$ is continuous for $t>s$;

It should also be mentioned that the above-mentioned proprieties were mainly established in $[1$, Theorem 2.3] and [50, Theorem 2.1], see also [3, 49].

One says that an evolution family $\mathcal{U}$ has an exponential dichotomy (or is hyperbolic) if there are projections $P(t)(t \in \mathbf{R})$ that are uniformly bounded and strongly continuous in $t$ and constants $\delta>0$ and $N \geq 1$ such that

(f) $U(t, s) P(s)=P(t) U(t, s)$;

(g) the restriction $U_{Q}(t, s): Q(s) \mathbf{X} \rightarrow Q(t) \mathbf{X}$ of $U(t, s)$ is invertible (we then set $\widetilde{U}_{Q}(s, t):=$ $\left.U_{Q}(t, s)^{-1}\right) ;$ and

(h) $\|U(t, s) P(s)\| \leq N e^{-\delta(t-s)}$ and $\left\|\widetilde{U}_{Q}(s, t) Q(t)\right\| \leq N e^{-\delta(t-s)}$ for $t \geq s$ and $t, s \in \mathbf{R}$.

According to [44], the following sufficient conditions are required for $A(t)$ to have exponential dichotomy.

(i) Let $(A(t), D(t))_{t \in \mathbf{R}}$ be generators of analytic semigroups on $\mathbf{X}$ of the same type. Suppose that $D(A(t)) \equiv D(A(0)), A(t)$ is invertible,

$$
\sup _{t, s \in \mathbf{R}}\left\|A(t) A(s)^{-1}\right\|
$$

is finite, and

$$
\left\|A(t) A(s)^{-1}-I\right\| \leq L_{0}|t-s|^{\mu}
$$

for $t, s \in \mathbf{R}$ and constants $L_{0} \geq 0$ and $0<\mu \leq 1$.

(j) The semigroups $\left(e^{\tau A(t)}\right)_{\tau \geq 0}, t \in \mathbf{R}$, are hyperbolic with projection $P_{t}$ and constants $N, \delta>0$. Moreover, let

$$
\left\|A(t) e^{\tau A(t)} P_{t}\right\| \leq \psi(\tau)
$$


and

$$
\left\|A(t) e^{\tau A_{Q}(t)} Q_{t}\right\| \leq \psi(-\tau)
$$

for $\tau>0$ and a function $\psi$ such that $\mathbf{R} \ni s \mapsto \varphi(s):=|s|^{\mu} \psi(s)$ is integrable with $L_{0}\|\varphi\|_{L^{1}(\mathbf{R})}<1$.

This setting requires some estimates related to $U(t, s)$. For that, we introduce the interpolation spaces for $A(t)$. We refer the reader to the following excellent books [4], [24], and [33] for proofs and further information on theses interpolation spaces.

Let $A$ be a sectorial operator on $\mathbf{X}$ (in assumption (H.1), replace $A(t)$ with $A$ ) and let $\alpha \in(0,1)$. Define the real interpolation space

$$
\mathbf{X}_{\alpha}^{A}:=\left\{x \in \mathbf{X}:\|x\|_{\alpha}^{A}:=\sup _{r>0}\left\|r^{\alpha}(A-\omega) R(r, A-\omega) x\right\|<\infty\right\},
$$

which, by the way, is a Banach space when endowed with the norm $\|\cdot\|_{\alpha}^{A}$. For convenience we further write

and

$$
\mathbf{X}_{0}^{A}:=\mathbf{X},\|x\|_{0}^{A}:=\|x\|, \mathbf{X}_{1}^{A}:=D(A)
$$

$$
\|x\|_{1}^{A}:=\|(\omega-A) x\| .
$$

Moreover, let $\hat{\mathbf{X}}^{A}:=\overline{D(A)}$ of $\mathbf{X}$. In particular, we have the following continuous embedding

$$
D(A) \hookrightarrow \mathbf{X}_{\beta}^{A} \hookrightarrow D\left((\omega-A)^{\alpha}\right) \hookrightarrow \mathbf{X}_{\alpha}^{A} \hookrightarrow \hat{\mathbf{X}}^{A} \hookrightarrow \mathbf{X}
$$

for all $0<\alpha<\beta<1$, where the fractional powers are defined in the usual way.

In general, $D(A)$ is not dense in the spaces $\mathbf{X}_{\alpha}^{A}$ and $\mathbf{X}$. However, we have the following continuous injection

$$
\mathbf{X}_{\beta}^{A} \hookrightarrow \overline{D(A)}\|\cdot\|_{\alpha}^{A}
$$

for $0<\alpha<\beta<1$

Given the family of linear operators $A(t)$ for $t \in \mathbf{R}$, satisfying (H.1), we set

$$
\mathbf{X}_{\alpha}^{t}:=\mathbf{X}_{\alpha}^{A(t)}, \quad \hat{\mathbf{X}}^{t}:=\hat{\mathbf{X}}^{A(t)}
$$

for $0 \leq \alpha \leq 1$ and $t \in \mathbf{R}$, with the corresponding norms. Then the embedding in Eq. (4.3) holds with constants independent of $t \in \mathbf{R}$. These interpolation spaces are of class $\mathcal{J}_{\alpha}$ ([33, Definition 1.1.1 ]) and hence there is a constant $c(\alpha)$ such that

$$
\|y\|_{\alpha}^{t} \leq c(\alpha)\|y\|^{1-\alpha}\|A(t) y\|^{\alpha}, \quad y \in D(A(t)) .
$$

We have the following fundamental estimates for the evolution family $\mathcal{U}$. Its proof was given in [7] though for the sake of clarity, we reproduce it here. 
Proposition 4.1. For $x \in \mathbf{X}, 0 \leq \alpha \leq 1$ and $t>s$, the following hold:

(i) There is a constant $c(\alpha)$, such that

$$
\|U(t, s) P(s) x\|_{\alpha}^{t} \leq c(\alpha) e^{-\frac{\delta}{2}(t-s)}(t-s)^{-\alpha}\|x\| .
$$

(ii) There is a constant $m(\alpha)$, such that

$$
\left\|\widetilde{U}_{Q}(s, t) Q(t) x\right\|_{\alpha}^{s} \leq m(\alpha) e^{-\delta(t-s)}\|x\|
$$

Proof. (i) Using (4.5) we obtain

$$
\begin{aligned}
\|U(t, s) P(s) x\|_{\alpha}^{t} & \leq c(\alpha)\|U(t, s) P(s) x\|^{1-\alpha}\|A(t) U(t, s) P(s) x\|^{\alpha} \\
& \leq c(\alpha)\|U(t, s) P(s) x\|^{1-\alpha}\|A(t) U(t, t-1) U(t-1, s) P(s) x\|^{\alpha} \\
& \leq c(\alpha)\|U(t, s) P(s) x\|^{1-\alpha}\|A(t) U(t, t-1)\|^{\alpha}\|U(t-1, s) P(s) x\|^{\alpha} \\
& \leq c(\alpha) N^{\prime} e^{-\delta(t-s)(1-\alpha)} e^{-\delta(t-s-1) \alpha}\|x\| \\
& \leq c(\alpha)(t-s)^{-\alpha} e^{-\frac{\delta}{2}(t-s)}(t-s)^{\alpha} e^{-\frac{\delta}{2}(t-s)}\|x\|
\end{aligned}
$$

for $t-s \geq 1$ and $x \in \mathbf{X}$.

Since $(t-s)^{\alpha} e^{-\frac{\delta}{2}(t-s)} \rightarrow 0$ as $t \rightarrow \infty$ it easily follows that

$$
\|U(t, s) P(s) x\|_{\alpha}^{t} \leq c(\alpha)(t-s)^{-\alpha} e^{-\frac{\delta}{2}(t-s)}\|x\| .
$$

If $0<t-s \leq 1$, we have

$$
\begin{aligned}
\|U(t, s) P(s) x\|_{\alpha}^{t} & \leq c(\alpha)\|U(t, s) P(s) x\|^{1-\alpha}\|A(t) U(t, s) P(s) x\|^{\alpha} \\
& \leq c(\alpha)\|U(t, s) P(s) x\|^{1-\alpha}\left\|A(t) U\left(t, \frac{t+s}{2}\right) U\left(\frac{t+s}{2}, s\right) P(s) x\right\|^{\alpha} \\
& \leq c(\alpha)\|U(t, s) P(s) x\|^{1-\alpha}\left\|A(t) U\left(t, \frac{t+s}{2}\right)\right\|^{\alpha}\left\|U\left(\frac{t+s}{2}, s\right) P(s) x\right\|^{\alpha} \\
& \leq c(\alpha) N e^{-\delta(t-s)(1-\alpha)} 2^{\alpha}(t-s)^{-\alpha} e^{-\frac{\delta \alpha}{2}(t-s)}\|x\| \\
& \leq c(\alpha) N e^{-\frac{\delta}{2}(t-s)(1-\alpha)} 2^{\alpha}(t-s)^{-\alpha} e^{-\frac{\delta \alpha}{2}(t-s)}\|x\| \\
& \leq c(\alpha) e^{-\frac{\delta}{2}(t-s)}(t-s)^{-\alpha}\|x\|,
\end{aligned}
$$

and hence

$$
\|U(t, s) P(s) x\|_{\alpha}^{t} \leq c(\alpha)(t-s)^{-\alpha} e^{-\frac{\delta}{2}(t-s)}\|x\| \text { for } t>s .
$$


(ii)

$$
\begin{aligned}
\left\|\widetilde{U}_{Q}(s, t) Q(t) x\right\|_{\alpha}^{s} & \leq c(\alpha)\left\|\widetilde{U}_{Q}(s, t) Q(t) x\right\|^{1-\alpha}\left\|A(s) \widetilde{U}_{Q}(s, t) Q(t) x\right\|^{\alpha} \\
& \leq c(\alpha)\left\|\widetilde{U}_{Q}(s, t) Q(t) x\right\|^{1-\alpha}\left\|A(s) Q(s) \widetilde{U}_{Q}(s, t) Q(t) x\right\|^{\alpha} \\
& \leq c(\alpha)\left\|\widetilde{U}_{Q}(s, t) Q(t) x\right\|^{1-\alpha}\|A(s) Q(s)\|^{\alpha}\left\|\widetilde{U}_{Q}(s, t) Q(t) x\right\|^{\alpha} \\
& \leq c(\alpha) N e^{-\delta(t-s)(1-\alpha)}\|A(s) Q(s)\|^{\alpha} e^{-\delta(t-s) \alpha}\|x\| \\
& \leq m(\alpha) e^{-\delta(t-s)}\|x\| .
\end{aligned}
$$

In the last inequality we have used that $\|A(s) Q(s)\| \leq c$ for some constant $c \geq 0$, see e.g. [46, Proposition 3.18].

In addition to above, we also need the following assumptions:

Hypothesis (H.2). The evolution family $\mathcal{U}$ generated by $A(\cdot)$ has an exponential dichotomy with constants $N, \delta>0$ and dichotomy projections $P(t)$ for $t \in \mathbf{R}$. Moreover, $0 \in \rho(A(t))$ for each $t \in \mathbf{R}$ and the following holds

$$
\sup _{t, s \in \mathbf{R}}\left\|A(s) A^{-1}(t)\right\|_{B\left(\mathbf{X}_{\alpha}, \mathbf{X}\right)}<c_{0} .
$$

Remark 4.2. Note that Eq. (4.8) is satisfied in many cases in the literature. In particular, it holds when $A(t)=d(t) A$ where $A: D(A) \subset \mathbf{X} \mapsto \mathbf{X}$ is any closed linear operator such that $0 \in \rho(A)$ and $d: \mathbf{R} \mapsto \mathbf{R}$ with $\inf _{t \in \mathbf{R}}|d(t)|>0$ and $\sup _{t \in \mathbf{R}}|d(t)|<\infty$.

Hypothesis (H.3). There exists $0 \leq \alpha<\beta<1$ such that

$$
\mathbf{X}_{\alpha}^{t}=\mathbf{X}_{\alpha} \text { and } \mathbf{X}_{\beta}^{t}=\mathbf{X}_{\beta}
$$

for all $t \in \mathbb{R}$, with uniform equivalent norms.

If $0 \leq \alpha<\beta<1$, then we let $k(\alpha)$ and $c^{\prime}$ denote respectively the bounds of the embedding $\mathbf{X}_{\beta} \hookrightarrow \mathbf{X}_{\alpha}$ and $\mathbf{X}_{\alpha} \hookrightarrow \mathbf{X}$, that is,

$$
\|u\|_{\alpha} \leq k(\alpha)\|u\|_{\beta}
$$

for each $u \in \mathbf{X}_{\beta}$ and

$$
\|u\| \leq c^{\prime}\|u\|_{\alpha}
$$

for each $u \in \mathbf{X}_{\alpha}$ 


\section{Main Results}

To study the existence and uniqueness of pseudo almost automorphic solutions to Eq. (1.1) we first introduce the notion of bounded solution.

Definition 5.1. A function $u: \mathbf{R} \mapsto \mathbf{X}_{\alpha}$ is said to be a bounded solution to Eq. (1.1) provided that the function $s \rightarrow A(s) U(t, s) P(s) f(s, B u(s))$ is integrable on $(-\infty, t)$, and the function $s \rightarrow$ $A(s) U(t, s) Q(s) f(s, B u(s))$ is integrable on $(t, \infty)$ for each $t \in \mathbb{R}$, and

$$
\begin{aligned}
u(t) & =-f(t, B u(t))-\int_{-\infty}^{t} A(s) U(t, s) P(s) f(s, B u(s)) d s \\
& +\int_{t}^{\infty} A(s) U(t, s) Q(s) f(s, B u(s)) d s+\int_{-\infty}^{t} U(t, s) P(s) g(s, C u(s)) d s \\
& -\int_{t}^{\infty} U(t, s) Q(s) g(s, C u(s)) d s
\end{aligned}
$$

for each $\forall t \in \mathbf{R}$.

Throughout the rest of the paper we denote by $\Gamma_{1}, \Gamma_{2}, \Gamma_{3}$, and $\Gamma_{4}$, the nonlinear integral operators defined by

$$
\begin{gathered}
\left(\Gamma_{1} u\right)(t):=\int_{-\infty}^{t} A(s) U(t, s) P(s) f(s, B u(s)) d s \\
\left(\Gamma_{2} u\right)(t):=\int_{t}^{\infty} A(s) U(t, s) Q(s) f(s, B u(s)) d s \\
\left(\Gamma_{3} u\right)(t):=\int_{-\infty}^{t} U(t, s) P(s) g(s, C u(s)) d s \\
\left(\Gamma_{4} u\right)(t):=\int_{t}^{\infty} U(t, s) Q(s) g(s, C u(s)) d s
\end{gathered}
$$

In this paper we suppose that the linear operators $B, C: \mathbf{X}_{\alpha} \mapsto \mathbf{X}$ are bounded and set

$$
\varpi:=\max \left(\|B\|_{B\left(\mathbf{X}_{\alpha}, \mathbf{X}\right)},\|C\|_{B\left(\mathbf{X}_{\alpha}, \mathbf{X}\right)}\right) .
$$

To study Eq. (1.1), in addition to the previous assumptions, we require that $p>1, \frac{1}{p}+\frac{1}{q}=1$, and that the following additional assumptions hold:

(H.4) $R(\omega, A(\cdot)) u \in A A\left(\mathbf{X}_{\alpha}\right)$ for each $u \in \mathbf{X}$. Moreover, for any sequence of real numbers $\left(\tau_{n}^{\prime}\right)_{n \in \mathbb{N}}$ there exist a subsequence $\left(\tau_{n}\right)_{n \in \mathbb{N}}$ and $G(\cdot, \cdot)$ such that

$$
G(t, s) P(s) u=\lim _{n \rightarrow \infty} A\left(s+\tau_{n}\right) U\left(t+\tau_{n}, s+\tau_{n}\right) P\left(s+\tau_{n}\right) u
$$

and

$$
A(s) U(t, s) P(s) u=\lim _{n \rightarrow \infty} G\left(t-\tau_{n}, s-\tau_{n}\right) P\left(s-\tau_{n}\right) u
$$

for all $t, s \in \mathbf{R}$ and $u \in \mathbf{X}_{\alpha}$. 
(H.5) Let $0 \leq \alpha<\beta<1$, and $f: \mathbf{R} \times \mathbf{X} \mapsto \mathbf{X}_{\beta}, g: \mathbf{R} \times \mathbf{X} \mapsto \mathbf{X}$ are $\mathbf{S}^{p}$-pseudo almost automorphic. Moreover, the functions $f, g$ are uniformly Lipschitz with respect to the second argument in the following sense: there exists $K>0$ such that

$$
\|f(t, u)-f(t, v)\|_{\beta} \leq K\|u-v\|,
$$

and

$$
\|g(t, u)-g(t, v)\| \leq K\|u-v\|
$$

for all $u, v \in \mathbf{X}$ and $t \in \mathbf{R}$.

The proof of our main result requires the following key technical Lemma.

Lemma 5.2. Under assumptions (H.1)-(H.3), then there exist constant $m(\alpha, \beta), n(\alpha)>0$ such that

$$
\begin{aligned}
& \left\|A(s) \widetilde{U}_{Q}(t, s) Q(s) x\right\|_{\alpha} \leq m(\alpha, \beta) e^{\delta(s-t)}\|x\|_{\beta} \quad \text { for } t \leq s \\
& \|A(s) U(t, s) P(s) x\|_{\alpha} \leq n(\alpha)(t-s)^{-\alpha} e^{-\frac{\delta}{2}(t-s)}\left\|_{x}\right\|_{\beta}, \quad \text { for } t>s .
\end{aligned}
$$

Proof. Let $x \in \mathbf{X}_{\beta}$. Since the restriction of $A(s)$ to $R(Q(s))$ is a bounded linear operator it follows that

$$
\begin{aligned}
\left\|A(s) \widetilde{U}_{Q}(t, s) Q(s) x\right\|_{\alpha} & \leq c k(\alpha)\left\|\widetilde{U}_{Q}(t, s) Q(s) x\right\|_{\beta} \\
& \leq c k(\alpha) m(\beta) e^{\delta(s-t)}\|x\| \\
& \leq m(\alpha, \beta) e^{\delta(s-t)}\|x\|_{\beta}
\end{aligned}
$$

for $t \leq s$ by using Eq. (4.7).

Similarly, for each $x \in \mathbf{X}_{\beta}$, using Eq. (4.8), we obtain

$$
\begin{aligned}
\|A(s) U(t, s) P(s) x\|_{\alpha} & =\left\|A(s) A(t)^{-1} A(t) U(t, s) P(s) x\right\|_{\alpha} \\
& \leq\left\|A(s) A(t)^{-1}\right\|_{B\left(\mathbf{X}_{\alpha}, \mathbf{X}\right)}\|A(t) U(t, s) P(s) x\|_{\alpha} \\
& \leq c_{0}\|A(t) U(t, s) P(s) x\|_{\alpha}
\end{aligned}
$$

for $t \geq s$.

First of all, note that $\|A(t) U(t, s)\| \leq K(t-s)^{-1}$ for all $t, s$ such that $0<t-s \leq 1$.

Now, let $t-s \geq 1$. Then, using Eq. (4.6), we obtain 


$$
\begin{aligned}
\|A(t) U(t, s) P(s) x\|_{\alpha} & =\|A(t) U(t, t-1) U(t-1, s) P(s) x\|_{\alpha} \\
& \leq\|A(t) U(t, t-1)\|_{B\left(\mathbf{X}_{\alpha}, \mathbf{X}\right)}\|U(t-1, s) P(s) x\|_{\alpha} \\
& \leq K c(\alpha) e^{-\frac{\delta}{2}(t-s)}(t-s)^{-\alpha}\|x\| \\
& \leq K K^{\prime} c(\alpha) e^{-\frac{\delta}{2}(t-s)}(t-s)^{-\alpha}\|x\|_{\alpha} \\
& \leq K K^{\prime} k(\alpha) c(\alpha) e^{-\frac{\delta}{2}(t-s)}(t-s)^{-\alpha}\|x\|_{\beta} \\
& \leq n^{\prime}(\alpha) e^{-\frac{\delta}{2}(t-s)}(t-s)^{-\alpha}\|x\|_{\beta} .
\end{aligned}
$$

Now, let $0<t-s \leq 1$. Again, using Eq. (4.6), we obtain

$$
\begin{aligned}
\|A(t) U(t, s) P(s) x\|_{\alpha} & =\left\|A(t) U\left(t, \frac{t+s}{2}\right) U\left(\frac{t+s}{2}, s\right) P(s) x\right\|_{\alpha} \\
& \leq\left\|A(t) U\left(t, \frac{t+s}{2}\right)\right\|_{B\left(\mathbf{X}_{\alpha}, \mathbf{X}\right)}\left\|U\left(\frac{t+s}{2}, s\right) P(s) x\right\|_{\alpha} \\
& \leq K c(\alpha) e^{-\frac{\delta}{4}(t-s)} 2^{\alpha}(t-s)^{-\alpha}\|x\| \\
& \leq K K^{\prime} c(\alpha) e^{-\frac{\delta}{4}(t-s)} 2^{\alpha}(t-s)^{-\alpha}\|x\|_{\alpha} \\
& \leq K K^{\prime} k(\alpha) c(\alpha) e^{-\frac{\delta}{4}(t-s)} 2^{\alpha}(t-s)^{-\alpha}\|x\|_{\beta} \\
& \leq n^{\prime \prime}(\alpha) e^{-\frac{\delta}{2}(t-s)}(t-s)^{-\alpha}\|x\|_{\beta} .
\end{aligned}
$$

Therefore,

$$
\|A(t) U(t, s) P(s) x\|_{\alpha} \leq n(\alpha) e^{-\frac{\delta}{2}(t-s)}(t-s)^{-\alpha}\|x\|_{\beta}
$$

for all $t, s \in \mathbf{R}$ with $t \geq s$.

Lemma 5.3. Under assumptions (H.1)-(H.5) and if

$$
N(\alpha, q, \delta):=\sum_{n=1}^{\infty}\left[\int_{n-1}^{n} e^{-q \frac{\delta}{2} s} s^{-q \alpha} d s\right]^{1 / q}<\infty,
$$

then the integral operators $\Gamma_{3}$ and $\Gamma_{4}$ defined above map $P A A\left(\mathbf{X}_{\alpha}\right)$ into itself.

Proof. Let $u \in P A A^{p}\left(\mathbf{X}_{\alpha}\right)$. Since $C \in B\left(\mathbf{X}_{\alpha}, \mathbf{X}\right)$ then $C u(\cdot) \in P A A^{p}(\mathbf{X})$. Setting $h(t)=$ $g\left(t, C(u(t))\right.$ and using the theorem of composition of $\mathbf{S}^{p}$-pseudo almost automorphic functions (Theorem 3.5) it follows that $h \in P A A^{p}(\mathbf{X})$. Now write $h=\phi+\zeta$ where $\phi^{b}$ belongs to $\left.A A\left(L^{p}(0,1), \mathbf{X}_{\alpha}\right)\right)$ and $\left.\zeta^{b} \in P A P_{0}\left(L^{p}(0,1), \mathbf{X}_{\alpha}\right)\right)$. Define for all $n=1,2, \ldots$, the sequence of integral operators 


$$
\Phi_{n}(t)=\int_{n-1}^{n} U(t, t-s) P(t-s) \phi(t-s) d s, \text { and } \Psi_{n}(t)=\int_{n-1}^{n} U(t, t-s) P(t-s) \zeta(t-s) d s
$$

for each $t \in \mathbf{R}$.

Letting $r=t-s$ it follows that

$$
\Phi_{n}(t)=\int_{t-n}^{t-n+1} U(t, r) P(r) \phi(r) d r
$$

and hence from the Hölder's inequality and the estimate Eq. (4.6) it follows that

$$
\begin{aligned}
\left\|\Phi_{n}(t)\right\|_{\alpha} & \leq \int_{t-n}^{t-n+1} c(\alpha) e^{-\frac{\delta}{2}(t-r)}(t-r)^{-\alpha}\|\phi(r)\| d r \\
& \leq c^{\prime} \int_{t-n}^{t-n+1} c(\alpha) e^{-\frac{\delta}{2}(t-r)}(t-r)^{-\alpha}\|\phi(r)\|_{\alpha} d r \\
& \leq c^{\prime} c(\alpha)\left[\int_{n-1}^{n} e^{-q \frac{\delta}{2} s} s^{-q \alpha} d s\right]^{1 / q}\|\phi\|_{\mathbf{S}^{p}} .
\end{aligned}
$$

From the assumption that $N(\alpha, q, \delta)$ is finite we then deduce from Weirstrass Theorem that the series $D(t):=\sum_{n=1}^{\infty} \Phi_{n}(t)$ is uniformly convergent on $\mathbf{R}$. Moreover, $D \in C\left(\mathbf{R}, \mathbf{X}_{\alpha}\right)$ and

$$
\|D(t)\|_{\alpha} \leq \sum_{n=1}^{\infty}\left\|\Phi_{n}(t)\right\|_{\alpha} \leq c^{\prime} c(\alpha) N(\alpha, q, \delta)\|\phi\|_{\mathbf{S}^{p}}
$$

for all $t \in \mathbf{R}$.

Let us show that $\Phi_{n} \in A A\left(\mathbf{X}_{\alpha}\right)$ for each $n=1,2,3, \ldots$ Indeed, since $\phi \in A S^{p}\left(\mathbf{X}_{\alpha}\right)$, for every sequence of real numbers $\left(\tau_{n}^{\prime}\right)_{n \in \mathbb{N}}$ there exist a subsequence $\left(\tau_{n_{k}}\right)_{k \in \mathbb{N}}$ and a function $\widehat{\phi}$ such that

$$
\int_{t}^{t+1}\left\|\widehat{\phi}(s)-\phi\left(s+\tau_{n_{k}}\right)\right\|_{\alpha}^{p} d s \rightarrow 0
$$

and

$$
\int_{t}^{t+1}\left\|\widehat{\phi}\left(s-\tau_{n_{k}}\right)-\phi(s)\right\|_{\alpha}^{p} d s \rightarrow 0
$$

as $k \rightarrow \infty$ pointwise in $\mathbf{R}$.

Define for all $n=1,2,3, \ldots$, the sequence of integral operators

$$
\widehat{\Phi}_{n}(t)=\int_{n-1}^{n} U(t, t-s) P(t-s) \widehat{\phi}(t-s) d s
$$

for all $t \in \mathbf{R}$.

Now 


$$
\begin{aligned}
\Phi\left(t+\tau_{n_{k}}\right)-\widehat{\Phi}(t) & =\int_{n-1}^{n} U\left(t, t+\tau_{n_{k}}-s\right) P\left(t+\tau_{n_{k}}-s\right) \phi\left(t+\tau_{n_{k}}-s\right) d s \\
& -\int_{n-1}^{n} U(t, t-s) P(t-s) \widehat{\phi}(t-s) d s \\
& =\int_{n-1}^{n} U\left(t, t+\tau_{n_{k}}-s\right) P\left(t+\tau_{n_{k}}-s\right) \phi\left(t+\tau_{n_{k}}-s\right) d s \\
& +\int_{n-1}^{n} U\left(t, t+\tau_{n_{k}}-s\right) P\left(t+\tau_{n_{k}}-s\right) \widehat{\phi}(t-s) d s \\
& -\int_{n-1}^{n} U\left(t, t+\tau_{n_{k}}-s\right) P\left(t+\tau_{n_{k}}-s\right) \widehat{\phi}(t-s) d s \\
& -\int_{n-1}^{n} U(t, t-s) P(t-s) \widehat{\phi}(t-s) d s \\
& =\int_{n-1}^{n} U\left(t, t+\tau_{n_{k}}-s\right) P\left(t+\tau_{n_{k}}-s\right)\left[\phi\left(t+\tau_{n_{k}}-s\right)-\widehat{\phi}(t-s)\right] d s \\
& +\int_{n-1}^{n}\left[U\left(t, t+\tau_{n_{k}}-s\right) P\left(t+\tau_{n_{k}}-s\right)-U(t, t-s) P(t-s)\right] \widehat{\phi}(t-s) d s .
\end{aligned}
$$

Using Lebesgue Dominated Convergence Theorem, one can easily see that

$\left\|\int_{n-1}^{n} U\left(t, t+\tau_{n_{k}}-s\right) P\left(t+\tau_{n_{k}}-s\right)\left[\phi\left(t+\tau_{n_{k}}-s\right)-\widehat{\phi}(t-s)\right] d s\right\|_{\alpha} \rightarrow 0$ as $k \rightarrow \infty, t \in \mathbf{R}$.

Similarly, using [9, Proposition 3.3] it follows that

$\left\|\int_{n-1}^{n}\left[U\left(t, t+\tau_{n_{k}}-s\right) P\left(t+\tau_{n_{k}}-s\right)-U(t, t-s) P(t-s)\right] \widehat{\phi}(t-s) d s\right\|_{\alpha} \rightarrow 0$ as $k \rightarrow \infty, t \in \mathbf{R}$.

Thus

$$
\widehat{\Phi}_{n}(t)=\lim _{k \rightarrow \infty} \Phi_{n}\left(t+\tau_{n_{k}}\right), \quad t \in \mathbf{R} .
$$

Similarly, one can easily see that

$$
\Phi_{n}(t)=\lim _{k \rightarrow \infty} \widehat{\Phi}_{n}\left(t-\tau_{n_{k}}\right)
$$

for all $t \in \mathbf{R}$ and $n=1,2,3, \ldots$ Therefore the sequence $\Phi_{n} \in A A\left(\mathbf{X}_{\alpha}\right)$ for each $n=1,2, \ldots$ and hence its uniform limit $D \in A A\left(\mathbf{X}_{\alpha}\right)$.

To complete the proof for $\Gamma_{3}$, we have to show that $\Psi_{n} \in P A P_{0}\left(\mathbf{X}_{\alpha}\right)$.

Letting $r=t-s$ it follows that

$$
\Phi_{n}(t)=\int_{t-n}^{t-n+1} U(t, r) P(r) \zeta(r) d r
$$


and hence from the Hölder's inequality and the estimate Eq. (4.6) it follows that

$$
\begin{aligned}
\left\|\Phi_{n}(t)\right\|_{\alpha} & \leq \int_{t-n}^{t-n+1} c(\alpha) e^{-\frac{\delta}{2}(t-r)}(t-r)^{-\alpha}\|\zeta(r)\| d r \\
& \leq c^{\prime} \int_{t-n}^{t-n+1} c(\alpha) e^{-\frac{\delta}{2}(t-r)}(t-r)^{-\alpha}\|\zeta(r)\|_{\alpha} d r \\
& \leq c^{\prime} c(\alpha)\left[\int_{n-1}^{n} e^{-q \frac{\delta}{2} s} s^{-q \alpha} d s\right]^{1 / q}\left[\int_{t-n}^{t-n+1}\|\zeta(s)\|_{\alpha}^{p} d s\right]^{1 / p},
\end{aligned}
$$

and hence $\Psi_{n} \in P A P_{0}\left(\mathbf{X}_{\alpha}\right)$ as $\zeta^{b} \in P A P_{0}\left(L^{p}\left((0,1), \mathbf{X}_{\alpha}\right)\right.$. From the assumption that $N(\alpha, q, \delta)$ is finite we then deduce from Weirstrass Theorem that the series $E(t):=\sum_{n=1}^{\infty} \Psi_{n}(t)$ is uniformly convergent on $\mathbf{R}$. Moreover, $E \in C\left(\mathbf{R}, \mathbf{X}_{\alpha}\right)$ and

$$
\|E(t)\|_{\alpha} \leq \sum_{n=1}^{\infty}\left\|\Phi_{n}(t)\right\|_{\alpha} \leq c^{\prime} c(\alpha) N(\alpha, q, \delta)\|\phi\|_{\mathbf{S}^{p}}
$$

for all $t \in \mathbf{R}$.

Consequently, the uniform limit $E(t)=\sum_{n=1}^{\infty} \Psi_{n}(t)$ belongs to $P A P_{0}\left(\mathbf{X}_{\alpha}\right)$. Therefore $\Gamma_{3}$ maps $P A A\left(\mathbf{X}_{\alpha}\right)$ into itself.

The proof for $\Gamma_{4}$ is similar to that of $\Gamma_{3}$ except that we make use of approximate Eq. (4.7) rather than Eq. (4.6).

Lemma 5.4. Under assumptions (H.1)-(H.5) and if

$$
N(\alpha, q, \delta):=\sum_{n=1}^{\infty}\left[\int_{n-1}^{n} e^{-q \frac{\delta}{2} s} s^{-q \alpha} d s\right]^{1 / q}<\infty
$$

then the integral operators $\Gamma_{1}$ and $\Gamma_{2}$ defined above map $P A A\left(\mathbf{X}_{\alpha}\right)$ into itself.

Proof. Let $u \in P A A^{p}\left(\mathbf{X}_{\alpha}\right)$. Since $B \in B\left(\mathbf{X}_{\alpha}, \mathbf{X}\right)$ then $B u(\cdot) \in P A A^{p}(\mathbf{X})$. Setting $h(t)=$ $f(t, B u(t))$ and using the theorem of composition of $\mathbf{S}^{p}$-pseudo almost automorphic functions (Theorem 3.5) it follows that $h \in P A A^{p}(\mathbf{X})$. Now write $h=\phi+\zeta$ where $\phi^{b}$ belongs to $\left.A A\left(L^{p}(0,1), \mathbf{X}_{\alpha}\right)\right)$ and $\left.\zeta^{b} \in P A P_{0}\left(L^{p}(0,1), \mathbf{X}_{\alpha}\right)\right)$. Define for all $n=1,2, \ldots$, the sequence of integral operators

$$
\Phi_{n}(t)=\int_{n-1}^{n} A(t-s) U(t, t-s) P(t-s) \phi(t-s) d s
$$

and

$$
\Psi_{n}(t)=\int_{n-1}^{n} A(t-s) U(t, t-s) P(t-s) \zeta(t-s) d s
$$


for each $t \in \mathbf{R}$.

Letting $r=t-s$ it follows that

$$
\Phi_{n}(t)=\int_{t-n}^{t-n+1} A(r) U(t, r) P(r) \phi(r) d r,
$$

and hence from the Hölder's inequality and the estimate Eq. (5.2) it follows that

$$
\begin{aligned}
\left\|\Phi_{n}(t)\right\|_{\alpha} & \leq \int_{t-n}^{t-n+1} n(\alpha) e^{-\frac{\delta}{2}(t-r)}(t-r)^{-\alpha}\|\phi(r)\|_{\beta} d r \\
& \leq c^{\prime} \int_{t-n}^{t-n+1} n(\alpha) e^{-\frac{\delta}{2}(t-r)}(t-r)^{-\alpha}\|\phi(r)\|_{\beta} d r \\
& \leq c^{\prime} n(\alpha)\left[\int_{n-1}^{n} e^{-q \frac{\delta}{2} s} s^{-q \alpha} d s\right]^{1 / q}\|\phi\|_{\mathbf{S}^{p}} .
\end{aligned}
$$

From the assumption that $N(\alpha, q, \delta)$ is finite we then deduce from Weirstrass Theorem that the series $D(t):=\sum_{n=1}^{\infty} \Phi_{n}(t)$ is uniformly convergent on $\mathbf{R}$. Moreover, $D \in C\left(\mathbf{R}, \mathbf{X}_{\alpha}\right)$ and

$$
\|D(t)\|_{\alpha} \leq \sum_{n=1}^{\infty}\left\|\Phi_{n}(t)\right\|_{\alpha} \leq c^{\prime} n(\alpha) N(\alpha, q, \delta)\|\phi\|_{\mathbf{S}^{p}}
$$

for all $t \in \mathbf{R}$.

Let us show that $\Phi \in A A\left(\mathbf{X}_{\alpha}\right)$. Indeed, since $\phi \in A S^{p}\left(\mathbf{X}_{\alpha}\right)$, for every sequence of real numbers $\left(\tau_{n}^{\prime}\right)_{n \in \mathbb{N}}$ there exist a subsequence $\left(\tau_{n_{k}}\right)_{k \in \mathbb{N}}$ and a function $\widehat{\phi}$ such that

$$
\int_{t}^{t+1}\left\|\widehat{\phi}(s)-\phi\left(s+\tau_{n_{k}}\right)\right\|_{\alpha}^{p} d s \rightarrow 0
$$

and

as $k \rightarrow \infty$ pointwise in $\mathbf{R}$.

$$
\int_{t}^{t+1}\left\|\widehat{\phi}\left(s-\tau_{n_{k}}\right)-\phi(s)\right\|_{\alpha}^{p} d s \rightarrow 0
$$

Define for all $n=1,2,3, \ldots$, the sequence of integral operators

$$
\widehat{\Phi}_{n}(t)=\int_{n-1}^{n} A(t-s) U(t, t-s) P(t-s) \widehat{\phi}(t-s) d s
$$

for all $t \in \mathbf{R}$.

Now

$$
\begin{aligned}
\Phi\left(t+\tau_{n_{k}}\right)-\widehat{\Phi}(t) & =\int_{n-1}^{n} A\left(t+\tau_{n_{k}}-s\right) U\left(t, t+\tau_{n_{k}}-s\right) P\left(t+\tau_{n_{k}}-s\right) \phi\left(t+\tau_{n_{k}}-s\right) d s \\
& -\int_{n-1}^{n} A(t-s) U(t, t-s) P(t-s) \widehat{\phi}(t-s) d s \\
& =I_{k, n}^{1}(t)+I_{k, n}^{2}(t),
\end{aligned}
$$


where

$$
I_{k, n}^{1}(t)=\int_{n-1}^{n} A\left(t+\tau_{n_{k}}-s\right) U\left(t, t+\tau_{n_{k}}-s\right) P\left(t+\tau_{n_{k}}-s\right)\left[\phi\left(t+\tau_{n_{k}}-s\right)-\widehat{\phi}(t-s)\right] d s
$$

and

$I_{k, n}^{2}(t)=\int_{n-1}^{n}\left[A\left(t+\tau_{n_{k}}-s\right) U\left(t, t+\tau_{n_{k}}-s\right) P\left(t+\tau_{n_{k}}-s\right)-A(t-s) U(t, t-s) P(t-s)\right] \widehat{\phi}(t-s) d s$.

Using Lebesgue Dominated Convergence Theorem, one can easily see that

$$
\left\|\int_{n-1}^{n} A\left(t+\tau_{n_{k}}-s\right) U\left(t, t+\tau_{n_{k}}-s\right) P\left(t+\tau_{n_{k}}-s\right)\left[\phi\left(t+\tau_{n_{k}}-s\right)-\widehat{\phi}(t-s)\right] d s\right\|_{\alpha} \rightarrow 0
$$

as $k \rightarrow \infty$ for each $t \in \mathbf{R}$.

Similarly, using (H.4) it follows that

$\left\|\int_{n-1}^{n}\left[A\left(t+\tau_{n_{k}}-s\right) U\left(t, t+\tau_{n_{k}}-s\right) P\left(t+\tau_{n_{k}}-s\right)-A(t-s) U(t, t-s) P(t-s)\right] \widehat{\phi}(t-s) d s\right\|_{\alpha} \rightarrow 0$ as $k \rightarrow \infty$ for each $t \in \mathbf{R}$.

Thus

$$
\widehat{\Phi}_{n}(t)=\lim _{k \rightarrow \infty} \Phi_{n}\left(t+\tau_{n_{k}}\right), \quad t \in \mathbf{R} .
$$

Similarly, one can easily see that

$$
\Phi_{n}(t)=\lim _{k \rightarrow \infty} \widehat{\Phi}_{n}\left(t-\tau_{n_{k}}\right)
$$

for all $t \in \mathbf{R}$ and $n=1,2,3, \ldots$ Therefore the sequence $\Phi_{n} \in A A\left(\mathbf{X}_{\alpha}\right)$ for each $n=1,2, \ldots$ and hence its uniform limit $E \in A A\left(\mathbf{X}_{\alpha}\right)$.

To complete the proof for $\Gamma_{1}$, we have to show that $\Psi_{n} \in P A P_{0}\left(\mathbf{X}_{\alpha}\right)$.

Letting $r=t-s$ it follows that

$$
\Phi_{n}(t)=\int_{t-n}^{t-n+1} A(r) U(t, r) P(r) \zeta(r) d r,
$$

and hence from the Hölder's inequality and the estimate Eq. (4.6) it follows that

$$
\begin{aligned}
\left\|\Phi_{n}(t)\right\|_{\alpha} & \leq \int_{t-n}^{t-n+1} n(\alpha) e^{-\frac{\delta}{2}(t-r)}(t-r)^{-\alpha}\|\zeta(r)\| d r \\
& \leq c^{\prime} \int_{t-n}^{t-n+1} n(\alpha) e^{-\frac{\delta}{2}(t-r)}(t-r)^{-\alpha}\|\zeta(r)\|_{\alpha} d r \\
& \leq c^{\prime} n(\alpha)\left[\int_{n-1}^{n} e^{-q \frac{\delta}{2} s} s^{-q \alpha} d s\right]^{1 / q}\left[\int_{t-n}^{t-n+1}\|\zeta(s)\|_{\alpha}^{p} d s\right]^{1 / p},
\end{aligned}
$$


and hence $\Psi_{n} \in P A P_{0}\left(\mathbf{X}_{\alpha}\right)$ as $\zeta^{b} \in P A P_{0}\left(L^{p}\left((0,1), \mathbf{X}_{\alpha}\right)\right.$. From the assumption that $N(\alpha, q, \delta)$ is finite we then deduce from Weirstrass Theorem that the series $E(t):=\sum_{n=1}^{\infty} \Psi_{n}(t)$ is uniformly convergent on $\mathbf{R}$. Moreover, $E \in C\left(\mathbf{R}, \mathbf{X}_{\alpha}\right)$ and

$$
\|E(t)\|_{\alpha} \leq \sum_{n=1}^{\infty}\left\|\Phi_{n}(t)\right\|_{\alpha} \leq c^{\prime} n(\alpha) N(\alpha, q, \delta)\|\phi\|_{\mathbf{S}^{p}}
$$

for all $t \in \mathbf{R}$. Consequently, the uniform limit $E(t)=\sum_{n=1}^{\infty} \Psi_{n}(t)$ belongs to $P A P_{0}\left(\mathbf{X}_{\alpha}\right)$. Therefore $\Gamma_{1}$ maps $P A A\left(\mathbf{X}_{\alpha}\right)$ into itself.

The proof for $\Gamma_{2} u(\cdot)$ is similar to that of $\Gamma_{1} u(\cdot)$ except that one makes use of Eq. (5.1) rather than Eq. (5.2).

Theorem 5.5. Suppose assumptions (H.1)-(H.5) hold. Moreover, suppose

$$
N(\alpha, q, \delta):=\sum_{n=1}^{\infty}\left[\int_{n-1}^{n} e^{-q \frac{\delta}{2} s} s^{-q \alpha} d s\right]^{1 / q}<\infty .
$$

Then the evolution equation (1.1) has a unique pseudo-almost automorphic mild solution whenever $K$ is small enough.

Proof. Consider the nonlinear operator $\mathbb{M}$ defined on $P A A\left(\mathbf{X}_{\alpha}\right)$ by

$$
\begin{aligned}
\mathbb{M} u(t) & =-f(t, B u(t))-\int_{-\infty}^{t} A(s) U(t, s) P(s) f(s, B u(s)) d s \\
& +\int_{t}^{\infty} A(s) U(t, s) Q(s) f(s, B u(s)) d s+\int_{-\infty}^{t} U(t, s) P(s) g(s, C u(s)) d s \\
& -\int_{t}^{\infty} U(t, s) Q(s) g(s, C u(s)) d s
\end{aligned}
$$

for each $t \in \mathbf{R}$.

As we have previously seen, for every $u \in P A A\left(\mathbf{X}_{\alpha}\right), f(\cdot, B u(\cdot)) \in P A A\left(\mathbf{X}_{\beta}\right) \subset P A A\left(\mathbf{X}_{\alpha}\right)$. In view of Lemma 5.3 and Lemma 5.4, it follows that $\mathbb{M}$ maps $P A A\left(\mathbf{X}_{\alpha}\right)$ into itself. To complete the proof one has to show that $\mathbb{M}$ has a unique fixed-point.

Let $v, w \in P A A\left(\mathbf{X}_{\alpha}\right)$. It is not difficult to see that

$$
\|\mathbb{M} v-\mathbb{M} w\|_{\infty, \alpha} \leq K \Theta \cdot\|v-w\|_{\infty, \alpha},
$$

where $\Theta:=\varpi\left[k(\alpha)+\delta^{-1}(m(\alpha, \beta)+m(\alpha))+(n(\alpha)+c(\alpha)) 2^{1-\alpha} \Gamma(1-\alpha) \delta^{\alpha-1}\right]$, and hence if $K$ is small enough, then Eq. (1.1) has a unique solution, which obviously is its only pseudo-almost automorphic solution. 
Example 5.6. Let $\Omega \subset \mathbf{R}^{N}(N \geq 1)$ be an open bounded subset with regular boundary $\Gamma=\partial \Omega$ and let $\mathbf{X}=L^{2}(\Omega)$ equipped with its natural topology $\|\cdot\|_{L^{2}(\Omega)}$.

Define the linear operator appearing in Eq. (1.2) as follows:

$$
A(t) u=a(t, x) \Delta u \text { for all } u \in D(A(t))=\mathbb{H}_{0}^{1}(\Omega) \cap \mathbb{H}^{2}(\Omega),
$$

where $a: \mathbf{R} \times \Omega \mapsto \mathbf{R}$ is a jointly continuous function, almost automorphic and satisfies the following assumptions:

$$
\inf _{t \in \mathbf{R}, x \in \Omega} a(t, x)=m_{0}>0, \text { and }
$$

(H.7) there exists $L>0$ and $0<\mu \leq 1$ such that

$$
|a(t, x)-a(s, x)| \leq L|s-t|^{\mu}
$$

for all $t, s \in \mathbf{R}$ uniformly in $x \in \Omega$.

First of all, note that in view of the above, $\sup _{t \in \mathbf{R}, x \in \Omega} a(t, x)<\infty$. Also, a classical example of a function $a$ satisfying the above-mentioned assumptions is for instance

$$
a_{\gamma}(t, x)=3+\sin |x| t+\sin \gamma|x| t,
$$

where $|x|=\left(x_{1}^{2}+\ldots+x_{N}^{2}\right)^{1 / 2}$ for each $x=\left(x_{1}, x_{2}, \ldots, x_{N}\right) \in \Omega$ and $\gamma \in \mathbf{R} \backslash \mathbb{Q}$.

Under previous assumptions, it is clear that the operators $A(t)$ defined above are invertible and satisfy Acquistapace-Terreni conditions. Moreover, it can be easily shown that

$$
R(\omega, a(\cdot, x) \boldsymbol{\Delta}) \varphi=\frac{1}{a(\cdot, x)} R\left(\frac{\omega}{a(\cdot, x)}, \boldsymbol{\Delta}\right) \varphi \in A A\left(\mathbf{X}_{1 / 2}\right)
$$

for each $\varphi \in L^{2}(\Omega)$ with

$$
\|R(\omega, a \boldsymbol{\Delta})\|_{B\left(L^{2}(\Omega)\right)} \leq \frac{\text { const. }}{|\omega|}
$$

Furthermore, assumptions (H.1)-(H.4) are fulfilled.

We require the following assumption:

(H.8) Let $\frac{1}{2}<\beta<1$, and $F, G: \mathbf{R} \times \mathbf{X}_{1 / 2} \mapsto \mathbf{X}_{\beta}$ be $S^{p}$-pseudo-almost automorphic functions in $t \in \mathbf{R}$ uniformly in $u \in \mathbf{X}_{1 / 2}$. Moreover, the functions $F, G$ are uniformly Lipschitz with respect to the second argument in the following sense: there exists $K^{\prime}>0$ such that

$$
\|F(t, \varphi)-F(t, \psi)\|_{\beta} \leq K^{\prime}\|\varphi-\psi\|_{L^{2}(\Omega)},
$$

and

$$
\|G(t, \varphi)-G(t, \psi)\|_{L^{2}(\Omega)} \leq K^{\prime}\|\varphi-\psi\|_{L^{2}(\Omega)}
$$

for all $\varphi, \psi \in L^{2}(\Omega)$ and $t \in \mathbf{R}$. 
We have

Theorem 5.7. Under previous assumptions including (H.6)-(H.8), then Eq. (1.2) has a unique solution $\varphi \in P A A\left(\mathbf{X}_{1 / 2}\right)$ whenever $K^{\prime}$ is small enough.

Classical examples of the above-mentioned functions $F, G: \mathbf{R} \times \mathbf{X}_{1 / 2} \mapsto L^{2}(\Omega)$ are given as follows:

$$
\begin{gathered}
F(t, \varphi)=\frac{K e(t)}{1+|\nabla \varphi|} \text { and } \\
G(t, \varphi)=\frac{K h(t)}{1+|\nabla \varphi|}
\end{gathered}
$$

where the functions $e, h: \mathbf{R} \mapsto \mathbf{R}$ are $\mathbf{S}^{p}$-pseudo-almost automorphic.

In this particular case, the corresponding reaction-diffusion equation, that is,

$$
\begin{cases}\frac{\partial}{\partial t}\left[\varphi+\frac{K e(t)}{1+|\nabla \varphi|}\right]=a(t, x) \Delta \varphi+\frac{K h(t)}{1+|\nabla \varphi|}, & \text { in } \mathbf{R} \times \Omega \\ \varphi=0, & \text { on } \mathbf{R} \times \Gamma\end{cases}
$$

has a unique solution $\varphi \in P A A\left(\mathbf{X}_{1 / 2}\right)$ whenever $K$ is small enough.

Received: October 2009. Revised: November 2009.

\section{References}

[1] P. Acquistapace, Evolution operators and strong solutions of abstract linear parabolic equations, Differential Integral Equations 1 (1988), 433-457.

[2] P. Acquistapace, F. Flandoli, B. Terreni, Initial boundary value problems and optimal control for nonautonomous parabolic systems. SIAM J. Control Optim. 29 (1991), 89-118.

[3] P. Acquistapace, B. Terreni, A unified approach to abstract linear nonautonomous parabolic equations, Rend. Sem. Mat. Univ. Padova 78 (1987), 47-107.

[4] H. Amann, Linear and Quasilinear Parabolic Problems, Birkhäuser, Berlin 1995.

[5] B. Amir and L. Maniar, Existence and some asymptotic behaviors of solutions to semilinear Cauchy problems with non dense domain via extrapolation spaces, Rend. Circ. Mat. Palermo (2000) 481-496.

[6] B. Amir and L. Maniar, Composition of Pseudo-Almost Periodic Functions and Cauchy Problems with Perator of Nondense Domain. Ann. Math. Blaise Pascal 6 (1999), no. 1, pp. $1-11$. 
[7] M. Baroun, S. Boulite, T. Diagana, and L. Maniar, Almost periodic solutions to some semilinear non-autonomous thermoelastic plate equations. J. Math. Anal. Appl. 349(2009), no. $1,74-84$.

[8] S. Bochner, Continuous mappings of almost automorphic and almost periodic functions, Proc. Nat. Acad. Sci. USA 52 (1964), pp. 907-910.

[9] M. Baroun, S. Boulite, G. M. N'Guérékata, And L. Maniar, Almost automorphy of Semilinear Parabolic Equations. Electron. J. Differential Equations 2008(2008), no. 60, 1-9.

[10] S. Boulite, L. Maniar, and G. M. N'Guérekata, Almost Automorphic Solutions for Hyperbolic Semilniear Evolution Equations, Semigroup Forum. Vol. 71 (2005), 231-240.

[11] D. Bugajewski And T. Diagana, Almost Automorphy of the Convolution Operator and Applications to Differential and Functional-Differential Equations, Nonlinear Stud. 13 (2006), no. 2, pp. 129-140.

[12] D. Bugajewski, T. Diagana, C. M. Mahop, Asymptotic and Pseudo Almost Periodicity of the Convolution Operator and Applications to Differential and Integral Equations. Z. Anal. Anwend. 25 (2006), no. 3, 327-340.

[13] P. Cieutat And K. Ezzinbi, Existence, uniqueness and attractiveness of a pseudo almost automorphic solutions for some dissipative differential equations in Banach spaces. J. Math. Anal. Appl. 354 (2009), no. 2, 494-506.

[14] G. Da Prato and P. Grisvard, Equations d'évolution abstraites non linéaires de type parabolique. Ann. Mat. Pura Appl. (4) 120 (1979) pp. 329-396.

[15] T. Diagana, Stepanov-like pseudo almost periodic functions and their applications to differential equations, Commun. Math. Anal. 3(2007), no. 1, pp. 9-18.

[16] T. Diagana, Stepanov-like pseudo almost periodicity and its applications to some nonautonmous differential Equations. Nonlinear Anal. 69 (2008), no. 12, 4277-4285.

[17] T. DiAgana, Existence of pseudo-almost automorphic solutions to some abstract differential equations with $\mathbf{S}^{p}$-pseudo-almost automorphic coefficients. Nonlinear Anal. 70 (2009), no. 11, 3781-3790.

[18] T. Diagana, Pseudo almost periodic functions in Banach spaces. Nova Science Publishers, Inc., New York, 2007.

[19] T. Diagana and E. Hernàndez M., Existence and Uniqueness of Pseudo Almost Periodic Solutions to Some Abstract Partial Neutral Functional-Differential Equations and Applications, J. Math. Anal. Appl. 327(2007), no. 2, pp. 776-791.

[20] T. Diagana, Existence and Uniqueness of Pseudo Almost Periodic Solutions to Some Classes of Partial Evolution Equations. Nonlinear Anal. 66 (2007), no. 2, 384-395. 
[21] T. Diagana and G. M. N'GuÉrékata, Pseudo Almost Periodic Mild Solutions To Hyperbolic Evolution Equationa in Abstract Intermediate Banach Spaces. Applicable Anal. 85 (2006), Nos. 6-7, pp. 769-780.

[22] T. Diagana, N. Henríquez, and E. Hernàndez, Almost automorphic mild solutions to some partial neutral functional-differential Equations and Applications. Nonlinear Anal. 69 (2008), no. 5, pp. 1485-1493.

[23] T. Diagana And G. M. N'GuÉrÉkata, Almost automorphic solutions to some classes of partial evolution equations. Appl. Math. Lett. 20 (2007), no. 4, pp. 462-466.

[24] K. J. Engel And R. Nagel, One Parameter Semigroups for Linear Evolution Equations, Graduate texts in Mathematics, Springer Verlag 1999.

[25] K. Ezzinbi, S. Fatajou and G. M. NGuÉrÉkata, Pseudo almost automorphic solutions to some neutral partial functional differential equations in Banach space. Nonlinear Anal. 70 (2009), no. 4, 1641-1647.

[26] K. Ezzinbi, S. Fatajou And G. M. NGuÉrÉkata, Pseudo almost automorphic solutions for dissipative differential equations in Banach spaces. J. Math. Anal. Appl. 351 (2009), no. $2,765-772$.

[27] A. M. Fink, Almost Periodic Differential Equations, Lecture Notes in Mathematics 377, Springer-Verlag, New York-Berlin, 1974.

[28] E. Hernández and H. R. Henríquez, Existence of Periodic Solutions of Partial neutral Functional Differential Equations with Unbounded Delay. J. Math. Anal. Appl 221 (1998), no. 2, pp. 499-522.

[29] E. Hernández, Existence Results for Partial Neutral Integro-differential Equations with Unbounded Delay. J. Math. Anal. Appl 292 (2004), no. 1, pp. 194-210.

[30] E. Hernández M., M. L. Pelicer, and J. P. C. dos Santos, Asymptotically Almost Periodic and Almost Periodic Solutions for a Class of Evolution Equations, Electron. J. Diff. Eqns 2004(2004), no. 61, pp. 1-15.

[31] Y. Hino, T. Naito, N. V. Minh, and J. S. Shin, Almost Periodic Solutions of Differential Equations in Banach Spaces. Stability and Control: Theory, Methods and Applications, 15. Taylor and Francis, London, 2002.

[32] J.-L. Lions and J. Peetre, Sur une classe d'espaces d'interpolation. Inst. Hautes tudes Sci. Publ. Math., no. 19 (1964), pp. 5-68.

[33] A. Lunardi, Analytic Semigroups and Optimal Regularity in Parabolic Problems, PNLDE Vol. 16, Birkhäauser Verlag, Basel, 1995. 
[34] J. Liang, J. Zhang, and T-J. Xiao, Composition of Pseudo Almost Automorphic and Asymptotically almost automorphic functions. J. Math. Anal. Appl. 340 (2008), no. 14931499.

[35] J. Liang, G. M. N'Guérékata, T-J. Xiao, And J. Zhang, Some properties of pseudo almost automorphic functions and applications to abstract differential equations. Nonlinear Anal. 70 (2009), no. 7, 2731-2735.

[36] L. Maniar, R. Schnaubelt, Almost periodicity of inhomogeneous parabolic evolution equations, Lecture Notes in Pure and Appl. Math. Vol. 234, Dekker, New York (2003), 299-318.

[37] M. G. Naso, A. Benabdallah, Thermoelastic plate with thermal interior control, Mathematical models and methods for smart materials (Cortona, 2001), 247-250, Ser. Adv. Math. Appl. Sci., 62, World Sci. Publ., River Edge, NJ, 2002.

[38] G. M. N'GuÉRÉKata, Almost automorphic functions and almost periodic functions in abstract spaces, Kluwer Academic / Plenum Publishers, New York-London-Moscow, 2001.

[39] G. M. N'GuÉrÉkata, Topics in almost automorphy, Springer, New york, Boston, Dordrecht, Lodon, Moscow 2005.

[40] G. M. N'GuÉrÉkata And A. Pankov, Stepanov-like almost automorphic functions and monotone evolution equations, Nonlinear Anal. 68 (2008), no. 9, pp. 2658-2667.

[41] A. Pankov, Bounded and almost periodic solutions of nonlinear operator differential equations, Kluwer, Dordrecht, 1990.

[42] A. PAZY, Semigroups of linear operators and applications to partial differential equations, Applied Mathematical Sciences, 44. Springer-Verlag, New York, 1983.

[43] J. Prüss, Evolutionary Integral Equations and Applications, Birkhäuser, 1993.

[44] R. Schnaubelt, Sufficient conditions for exponential stability and dichotomy of evolution equations, Forum Math. 11(1999), 543-566.

[45] R. Schnaubelt, Asymptotically autonomous parabolic evolution equations, J. Evol. Equ. 1 (2001), 19-37.

[46] R. Schnaubelt, Asymptotic behavior of parabolic nonautonomous evolution equations, in: M. Iannelli, R. Nagel, S. Piazzera (Eds.), Functional Analytic Methods for Evolution Equations, in: Lecture Notes in Math., 1855, Springer-Verlag, Berlin, 2004, 401-472.

[47] T-J. Xiao, J. Liang, J. Zhang, Pseudo almost automorphic solutions to semilinear differential equations in Banach spaces. Semigroup Forum 76 (2008), no. 3, 518-524.

[48] Ti-J. Xiao, X-X. Zhu, J. Liang, Pseudo-almost automorphic mild solutions to nonautonomous differential equations and applications. Nonlinear Anal. 70 (2009), no. 11, 40794085 . 
[49] A. YAGI, Parabolic equations in which the coefficients are generators of infinitely differentiable semigroups II, Funkcial. Ekvac. 33 (1990), 139-150.

[50] A. YAGI, Abstract quasilinear evolution equations of parabolic type in Banach spaces, Boll. Un. Mat. Ital. B (7) 5 (1991), 341-368.

[51] S. Zaidman, Topics in Abstract Differential Equations, Pitman Research Notes in Mathematics Ser. II John Wiley and Sons, New York, 1994-1995. 